\title{
Los pronósticos matemáticos son hipótesis: consideración de la incertidumbre en la presentación de datos sobre la pandemia por COVID-19
}

\section{Mathematical Forecasts are hypotheses: considering uncertainty in publishing COVID-19 pandemic data}

Rubén Aroca Jácomea

a Facultad de Filosofía, Universidad Católica de Santiago de Guayaquil, Ecuador

\section{Resumen}

La época actual es, a un tiempo, de elevada incertidumbre y producción de información. En la estimación de los escenarios producidos por la pandemia por COVID-19, se construyen pronósticos producidos por modelos matemáticos que contemplan determinados niveles de error. Esto lleva a reconsiderar la forma usual de tratamiento de datos, tanto en el periodismo como en la investigación académica, produciendo nuevos énfasis sobre la necesidad de exhaustivos exámenes de la información disponible, pues con frecuencia los especialistas no son lo suficientemente claros en sus parámetros o supuestos de partida, omisiones que adoptan otras dimensiones en los medios de comunicación, pues los periodistas suelen asumir frente a información sensible las mismas actitudes que con relación a la información producida en "periodos de normalidad". En este marco, se considera necesario la formación de una comprensión adecuada de la data sobre contagios, defunciones o medidas orientadas a la contención del COVID-19, considerándose útil la formulación de reglas básicas que tanto especialistas como periodistas puedan tener en cuenta a la hora de comunicar resultados o publicar noticias en un tiempo tan crítico como el de la pandemia.

Palabras clave: Pandemia por COVID-19; pronósticos matemáticos; periodismo; investigación académica.

\begin{abstract}
The current period is, at the same time, highly uncertainty and intensive in data production. In the analysis of scenarios produced by the COVID-19 pandemic, we make forecast based on mathematical models where exact assumptions are never true. This leads to a reconsideration of the usual form of data analysis, both in journalism and academic research, bolding on the need for exhaustive testing of the available information, since researchers are often not clear enough in their parameters or assumptions. These omissions usually become in different levels of importance in the media, because journalists tend to assume the same attitudes towards complex data just in the way they do with the news in "normal periods".

However, it is necessary to obtain an adequate understanding of the data on positive COVID-19 cases, defunctions or politics aimed to containing the virus, designing some basic rules that both specialists and journalists may take into account when they have to write papers or publish news at a time as critical as nowadays.
\end{abstract}

Keywords: COVID-19 pandemic; mathematical forecast models; journalism; academic research. 
"In God we trust. All others must bring data" Deming, $W$

\section{Introducción}

ए I presente artículo es motivado por dos propósitos iniciales: 1) la necesidad de entender la naturaleza y las limitaciones de las estimaciones matemáticas y estadísticas, en especial cuando estas se vuelven un objeto noticiable (como en el caso de la información sobre el crecimiento de contagios y muertes por COVID-19); y, 2) la necesidad de diseñar ciertas indicaciones básicas para facilitar la comunicación de información sensible acerca de la situación referida a la pandemia.

\section{Algunos antecedentes}

El 16 de marzo de 2020, al inicio del estado de excepción para combatir la propagación del COVID-19, el presidente del Ecuador indicó que se podría llegar en el país a 800.000 contagiados, cita que fue repetida y no examinada por todos los medios.

El 26 de marzo, el Diario Expreso publicó las estimaciones de contagio y requerimiento de servicios hospitalarios realizadas por una empresa española, estudio que sostenía que en Madrid pudiera haber medio millón de contagiados para ese entonces (Moncada, 2020). La empresa que realizó las proyecciones no contaba con especialización en epidemiología 0 afines, y en España, a pesar de lo difícil de escenario actual, no se ha observado niveles de contagio de esas proporciones (Bort, 2020).

Los casos anotados no solo permiten observar que los pronósticos producidos por modelos matemáticos pueden contemplar ciertos niveles de error, sino que, en ocasiones su uso es simplemente fallido y contraproducente.

En períodos críticos, la información debe ser exhaustivamente examinada. Con frecuencia los especialistas no son lo suficientemente claros en sus parámetros o supuestos de partida y, en lo posterior, estas omisiones adoptan otras dimensiones en los medios de comunicación, pues los periodistas suelen asumir frente a información sensible las mismas actitudes que con relación a la información producida en "periodos de normalidad". Citar declaraciones de cualquier funcionario o científico, sin mediar un adecuado examen de datos, no constituye (al menos, no en estos casos) un papel destacable en la construcción de opinión fundamentada sino absolutamente todo lo contrario.

En este marco, se considera necesario la formación de una comprensión adecuada de la información, de sus potencialidades y limitaciones y, claro está, de su relación con la realidad. Para ello, resulta útil formular algunas recomendaciones que tanto especialistas como periodistas puedan tener en cuenta a la hora de comunicar resultados o publicar noticias en un tiempo tan crítico como el de la pandemia por COVID-19. Estas recomendaciones se basan especialmente en: Goodson (2020), Ordway (2020), Red Etica (2020) y Paullus (2000).

Los componentes de la información sobre COVID-19

A mediados de febrero de 2020, en medio de purgas internas en la cúpula provincial del partido por sospechas de un manejo inadecuado de la crisis, en la provincia Hubei se modificó el criterio de detección de nuevos casos de infección por COVID-19 mediante la inserción de una nueva categoría denominada "casos clínicos". En resumidos términos, esto implicó contabilizar como casos no sólo aquellos en los que practicaba el test para la detección del virus, sino también a todos aquellos que presentaban los síntomas característicos (Feng, 2020). El resultado de dicha decisión hizo que Hubei pase de un reporte de 1.638 casos positivos el 11 de febrero a otro con 14.840 casos positivos al siguiente día (Gráfico 1).

El efecto en la representación estadística (Gráfico 1) puede describirse como un "disparo" del tamaño de la población contagiada que al publicarse tal cual, por los servicios de noticias, implicó la producción de una mayor inquietud en los públicos interesados. No obstante, lo que había variado era solamente una definición conceptual respecto a qué considerar un "caso real" de contagio, y no la realidad del contagio en sí. 


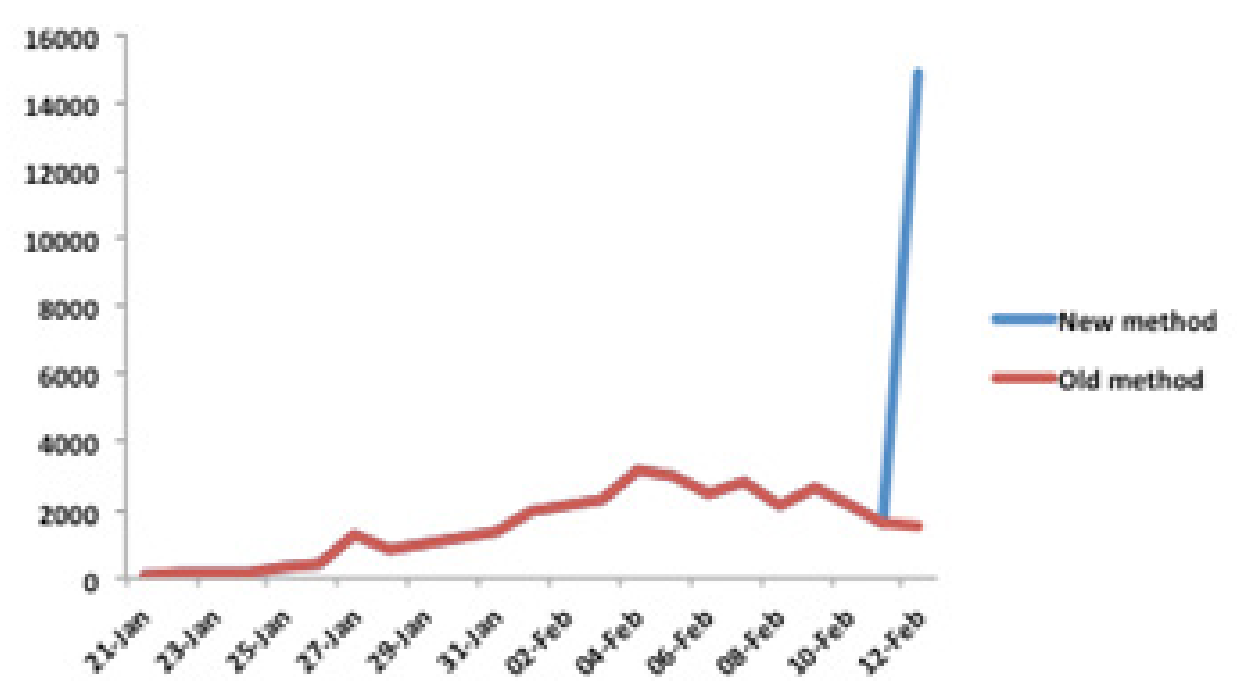

Gráfico 1. Hubei. Reporte diario de casos confirmados positivos por COVID 19. Fuente: Feng, 2020.

En una buena parte de los países de occidente, "se considera como caso diagnosticado como positivo a todo caso confirmado por laboratorio, independiente de los signos clínicos o síntomas" (Ministerio de Sanidad - Gobierno de España, 2020). Esta es la situación de países como España, Colombia o Ecuador. Sin embargo, los datos podrían modificarse también si consideramos, tal como lo hizo la provincia de Hubei en su momento, las siguientes definiciones (Organización Mundial de la Salud, 2020):

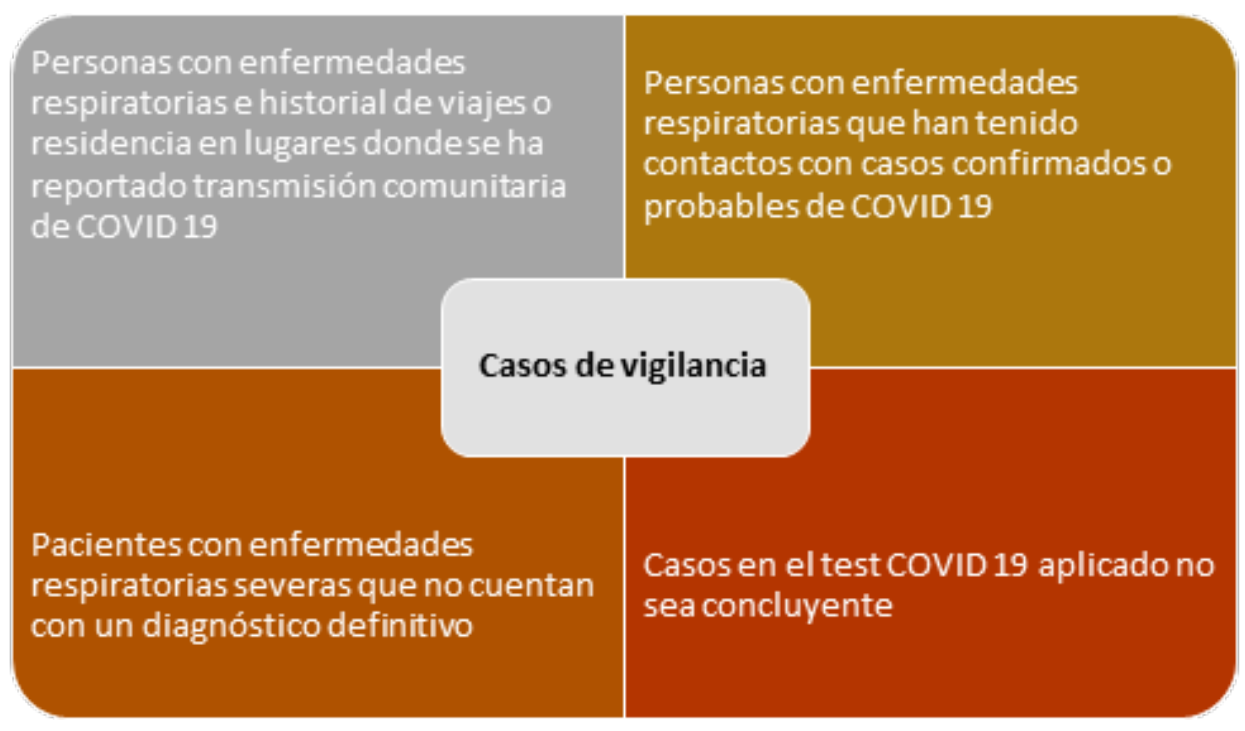

Ilustración 1. Definiciones de casos para vigilancia. Guidance on global surveillance for COVID-19, WHO, 27 February 2020. 
Cualquiera de las cuatro tipologías descritas (llustración 1) implican, por un lado, el desarrollo de procesos de observación y registro que probablemente no siempre se ajustan a las recomendaciones de la Organización Mundial de la Salud (OMS); y, en segundo lugar, la transformación de la información que representa el crecimiento diario o periódico de los casos de COVID-19. Realizar estas estimaciones toma tiempo e integran, claro está, un cierto nivel de incertidumbre.

La OMS ha solicitado a los organismos competentes delárea de salud en cada país, la generación de un reporte confirmatorio de casos de COVID-19 dentro de las 48 horas siguientes a su detección. El reporte se compone de un total de 82 campos o variables que requieren de un seguimiento detallado de cada paciente y de personal adiestrado para el registro de la información.

La producción de datos sobre el crecimiento del contagio y su letalidad resulta, por tanto, un problema aparte. Es comprensible que entre los tiempos de aplicación de test, la obtención de los resultados, su validación y registro de información sumen varios días o semanas hasta su publicación. En el caso de Gran Bretaña, se estima que este proceso puede tomar hasta 4 (Doyle, 2020) días mientras que en Colombia puede tomar entre dos y tres semanas (López, 2020).

En conclusión, en primer lugar, los reportes diarios que emiten los países no representan el crecimiento que el contagio adquiere de un día al siguiente, ni la forma natural en que la epidemia se comporta. Representa, eso sí, el esfuerzo de cada país tanto por otorgar respuestas ante el fenómeno como por registrar dicho proceso. Y es justo aquí donde incertidumbre y estadísticas cobran una mayor importancia.

En segundo lugar, toda proyección estadística del número de casos infectados por COVID-19 tiene sesgos que dependen de: número de test aplicados en cada país; tiempo transcurrido entre la detección de un caso y su ingreso al sistema de información nacional correspondiente; y el tiempo transcurrido entre la situación descrita y su comunicación a sus distintos usuarios.

\section{Presentando información crítica: algunas conclusiones y recomendaciones}

Las recomendaciones que se incluyen a continuación se formulan en consideración de la necesidad de entender la naturaleza y las limitaciones de las estimaciones matemáticas y estadísticas, en la perspectiva de facilitar la comunicación de información sensible acerca de la pandemia por COVID-19. Estas reglas, que pretenden encontrar utilidad cuando se trate de comunicar datos 0 publicar noticias en un tiempo tan crítico como el presente, consideran que sus usuarios potenciales son tanto los investigadores especialistas como los informadores de medios periodísticos.

Primera recomendación: sin indicaciones sobre su procedencia, es imposible conocer la validez estadística. Corrija, por tanto, la abstracción desmesurada de las representaciones estadísticas

Desconocemos el contexto de casi todas las estadísticas que leemos u oímos comentar, y esto implica no solo a quienes se supone que las estadísticas representan sino también a quienes las construyen, a sus antecedentes y procedimientos. Sin una ambientación, sin un trasfondo y sin indicaciones sobre su procedencia es imposible conocer la validez estadística. Por otro lado, en literatura o en narrativa, el foco de atención se concentra casi siempre en los individuos y no en el análisis, las argumentaciones y las medidas: es decir, casi siempre es concreto y rara vez abstracto. Un enfoque así es necesario para corregir la abstracción desmesurada y mantener la estadística dentro de la perspectiva humana.

Segunda recomendación: los modelos funcionan, en cierto modo, a la manera de hipótesis. Por tanto, científicos y periodistas deben expresar el nivel de incertidumbre asociado al pronóstico

Todo pronóstico matemático se formula en calidad de hipótesis, la misma que posee un componente de incertidumbre asociado a la proyección o pronóstico. Una hipótesis es una afirmación con cierta probabilidad de ocurrir, pero lo que hace que una hipótesis sea considerada como tal es que su probabilidad de ocurrencia es indeterminada. La indeterminación o incertidumbre puede formularse de varios modos, ya sea como rango de error o como la forma en que deben ser consideradas las afirmaciones del pronóstico.

Tomemos en consideración el siguiente ejemplo. La estimación anterior (llustración 2) calcula el nivel de contagio por COVID-19 no a través de la progresión de casos diarios, sino a través de la tasa de defunción. Esto parte de considerar como supuesto hallazgos de ciertos estudios (Wu \& McGoogan, 2020) que sitúan la mortalidad por COVID-19 ente el $2 \%$ y $3 \%$ de los casos de contagio. Este sería la base del pronóstico y, como tal, puede resultar muy cuestionable. 


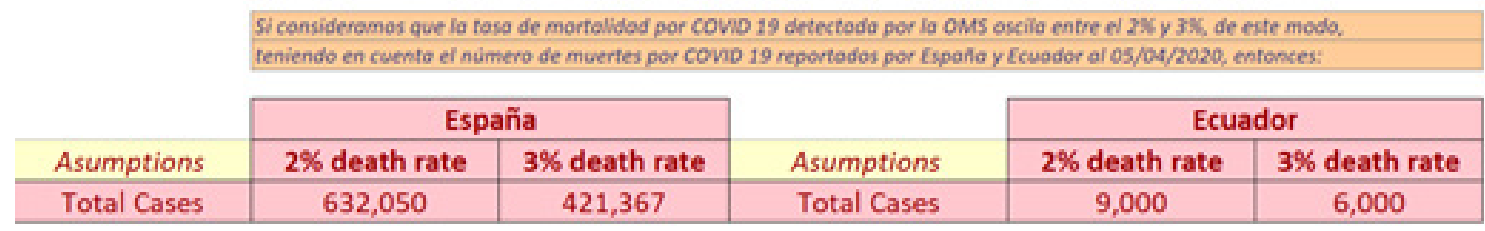

Ilustración 2. Consideración de la tasa de mortalidad como supuesto para estimar nivel de contagio de la población.

Tercera recomendación: escoja cuidadosamente los expertos a los que consulta

Todos tenemos o experimentamos la ansiedad por acceder a información adecuada. En las redacciones, esta necesidad puede sentirse como apremio, presión por la competencia, etc. Esto puede producir que periodistas urgidos por el cierre de la edición pregunten a profesionales no competentes dejándose guiar por criterios poco pertinentes y obtengan, por este vía, opiniones inexactas o inválidas. Esto es particularmente crítico cuando se trata de temas de salud pública.

Antes de seleccionar a un experto, es necesario revisar su experiencia o lo que ha escrito respecto al tema objeto de análisis o su vinculación a un área muy relacionada a dicho objeto. Una vez que haya lo grado dar este paso, recuerde: un experto expone sus pronósticos o los resultados de su trabajo, pero los mismos deben ser sometidos a observación de otros.

Cuarta recomendación: los periodistas deben consultar y citar otros expertos antes de publicar un pronóstico

Con referencia a lo anterior, los resultados producidos por la aplicación de un modelo deben ser sometidos a evaluación de especialistas, pues es de este modo en que se puede dejar en claro la actitud que se debe asumir frente a dichos resultados. Debe evitarse relacionar estos productos con explicaciones basadas en opiniones o con nociones del sentido común.

Quinta recomendación: los científicos e investigadores deben describir con claridad los parámetros críticos y supuestos que utilizan en sus modelos o pronósticos. De lo contrario, usted tendrá que hacer que los pongan en claro

¿Cuáles son las condiciones que deben cumplirse para que los pronósticos obtenidos por la aplicación de un modelo puedan considerarse como probables? Si la tasa de contagio por COVID-19 está en función tanto de la capacidad de transmisión del virus en sí, como de la efectividad de las políticas de aislamiento y capacidad hospitalaria, es necesario comprender el rango en que juegan estos supuestos.

Es muy comentado, por estos tiempos, el caso del tratamiento que el diario Financial Times hizo de la presentación del modelo producido a partir del paper conocido como Estudio Oxford. Este modelo, desarrollado por el Imperial College de Londres, describe una forma de actuación implementada en la mayor parte de las naciones de occidente para "aplanar la curva" (Ferguson et al., 2020), que consiste en imponer medidas de alejamiento social más extremas cada vez que los ingresos en las unidades de cuidados intensivos (UCl) empiezan a aumentar, y suavizarlas al reducirse la cantidad de las personas ingresadas. Parte del funcionamiento del modelo matemático implica considerar escenarios de alto contagio y bajo contagio de la población. El diario Financial Times (llustración 3) tomó dichos parámetros como realidades potenciales e informó que la mitad de la población del Reino Unido se encontraba "ya contagiada" (Cookson, 2020).

En el caso anterior, lo que correspondía en primer lugar era una revisión exhaustiva de los supuestos y componentes del modelo e informar en qué consistían y estimar qué tan lejos o distante se encontraba la población de dicho escenario. En segundo lugar, habría sido de gran utilidad relacionar estos resultados con la aplicación de otros modelos matemáticos sobre el problema. En este caso, las analogías con simulaciones realizadas en otros países, como China, podrían haber resultado útiles (Trilla, 2020).

\section{Sexta recomendación: ser lo más transparente posible}

Si se va a hablar de los resultados de la aplicación de un modelo o una proyección estadística, todo el modelo 0 proyección, así como sus hojas de datos, estructura de cálculo o códigos, deben ser de acceso libre (llustración 4). Esto implica una exigencia doble: por un lado, que el especialista esté dispuesto a poner a disposición sus datos para que otros pueden examinarlos y, por otro, que los periodistas tengan la oportunidad de también recoger los criterios derivados de evaluaciones de pares. 


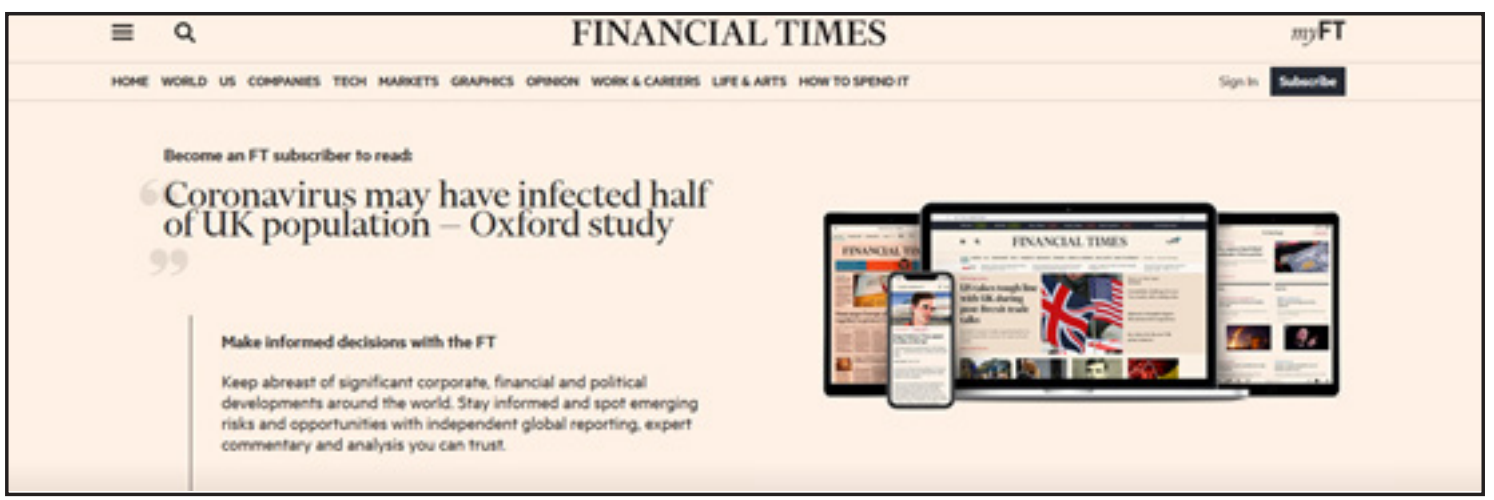

Ilustración 3. Sección de la nota del diario FT. Véase: https://www.ft.com/content/5ff6469a-6dd8-11ea-89df41 bea055720b

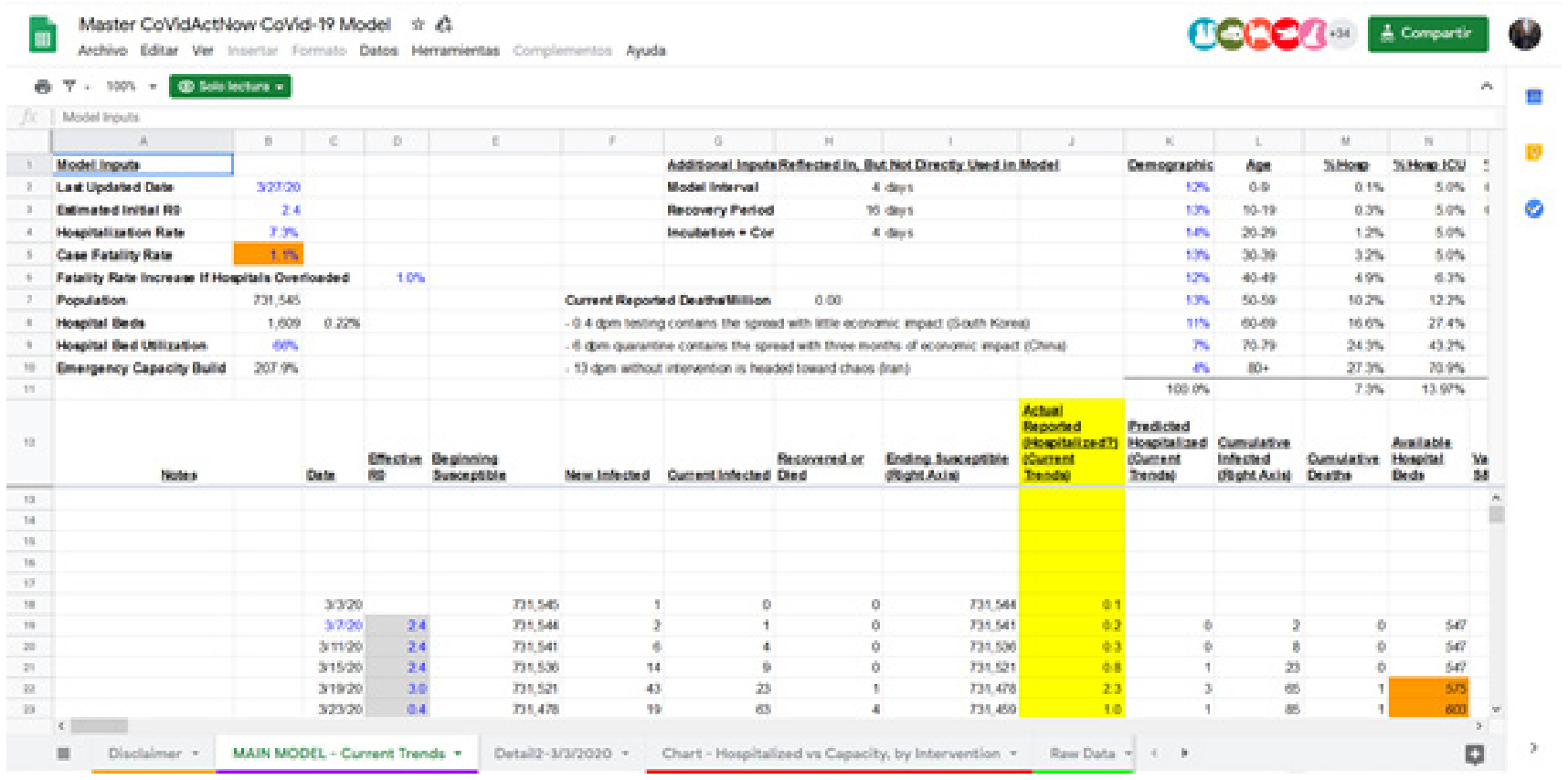

Ilustración 4. Modelo Master CoVidActNow CoVid-19 Model, totalmente liberado. Véase: https://docs.google.com/ spreadsheets/d/1YEj4Vr6IG1jQ1R3LG6frijJYNynKcgTjzo2n0FsBwZA/edit\#gid=1579455912

Séptima recomendación: los tomadores de decisión deben utilizar múltiples modelos para fundamentar el diseño de una política

Como se indicó en el apartado 5, al hablar de las medidas que toman para enfrentar la pandemia por COVID-19, varios gobiernos argumentan desde el modelo desarrollado por el Imperial College (Ferguson et al., 2020). Sin embargo, se conoce bien de la existencia de otros modelos (CoVidActNow, 2020). Es pertinente, por tanto, preguntar en qué medida se ha procedido a comparar los modelos existentes, sus resultados y su congruencia.

Tomar decisiones sobre la consideración de un solo modelo de pronóstico no sólo que no resulta científica y técnicamente razonable, sino que, en este caso, puede hacer la diferencia en la tasa de contagio y defunciones (llustración 3).

Octava recomendación: indicar cuando el modelo o pronóstico es producido por una fuente sin formación en enfermedades infecciosas

Un centro de investigaciones especializado en modelos generados por inteligencia artificial o redes neuronales de análisis puede propiciar nuevos recursos para procesar la información, incluso si los resultados de sus estudios no resultan plausibles. Por ello, si se está frente a un modelo diseñado por un organismo o universidad sin experiencia en epidemiología 0 enfermedades 
infecciones, es necesario tener una doble actitud. Por un lado, poner los resultados de ese ejercicio bajo escrutinio de los epidemiólogos de modo que sea adecuadamente valorado, pero asegurando, por otro lado, brindar oportunidad suficiente a otros ángulos de análisis científico que podrían resultar de importancia.

\section{Conclusiones}

El presente artículo partió de haber identificado la necesidad de: 1) entender la naturaleza y las limitaciones de las estimaciones matemáticas y estadísticas; y 2) diseñar, en consecuencia, ciertas reglas básicas para facilitar la comunicación de información sensible acerca de la situación referida a la pandemia por COVID-19.

Se considera que sin indicaciones sobre la forma de producción de datos y procedencia, los usuarios de la información (gestores de salud, periodistas, políticos y públicos) no podrán determinar: a) el nivel de validez asociado a la estimación, cuestión que se debe tener a cuenta en la perspectiva de corregir la abstracción desmesurada y mantener la estadística dentro de la perspectiva humana; b) el componente de incertidumbre metodológicamente asociado al pronóstico matemático, el mismo que generalmente se formula como rango de error o como en relación a determinadas condicionalidades.

Esto no suele considerarse adecuadamente, generalmente por omisiones expositivas de tipo metodológico o por el apremio por el cierre de la edición. Esto es particularmente crítico cuando se trata de temas de salud pública. Por ello, debe comprenderse que, una vez que los expertos han expuesto sus pronósticos 0 los resultados de su trabajo, éstos deben ser sometidos a observación de otros, en la perspectiva de dejar en claro la actitud que se debe asumir frente a dichos resultados. Como condición adicional, se recomienda que todo el modelo o proyección, así como sus hojas de datos, estructura de cálculo o códigos, deben ser liberadas para observación.

La aplicación de las medidas enunciadas produce la posibilidad de comparar varios modelos existentes, sus resultados y congruencia, en la perspectiva de evitar la toma de decisiones sesgadas por los outputs de un solo modelo de pronóstico. El impacto de esta actitud puede hacer la diferencia en los ritmos de la tasa de contagio y defunciones.

Finalmente, se sostiene que las reglas enunciadas no deben inhibir al investigador o informador en preservar su apertura frente a fuentes emergentes de producción de datos, suponiendo, por un lado, que los resultados de ese ejercicio deben situarse bajo escrutinio especializado, pero asegurando, por otro lado, oportunidad suficiente a una pluralidad de ángulos de análisis.

Agradecimientos

Extiendo mi agradecimiento al Instituto de Investigación e Innovación en Ciencias Sociales, Estudios Humanísticos y Arte de la Universidad Católica de Santiago de Guayaquil.

\section{Referencias bibliográficas}

Bort, C. (2020, 22 de marzo). Coronavirus: algoritmos para dimensionar los recursos sanitarios. Estamos a tiempo. Recuperado de Medium: https:// medium.com/@carlosbort/coronavirus-algoritmospara-dimensionar-los-recursos-sanitarios-estamosa-tiempo-98e4df7f59b8

Cookson, C. (2020, 24 de marzo). Coronavirus may have infected half of UK population - Oxford study. Recuperado de Financial Times: https:// www.ft.com/content/5ff6469a-6dd8-11ea-89df41bea055720b

CoVidActNow. (2020, 27 de marzo). COVID-19 Data Model. Recuperado de Master CoVidActNow CoVid-19 Mode: https://docs.google.com/spreadsheets/d/1Y Ej4Vr6IG1jQ1R3LG6frijJYNynKcgTjzo2nOFsBwZA/ edit\#gid=1579455912

Diario Expreso. (2020, 26 de marzo). Actualidad. Recuperado de Expreso.ec: https://www.Expreso. ec/actualidad/coronavirus-ecuador-carlos-bortestadisticas-contagios-7860.html

Doyle, L. (2020, 7 de abril). Coronavirus test: How long does it take to get your test back? Recuperado deExpress: https://www.express.co.uk/life-style/ health/1253887/coronavirus-test-how-long-to-getcoronavirus-test-back.

Feng, E.(2020,13defebrero). AChange In How1 Chinese Province Reports Coronavirus Adds Thousands Of Cases. Recuperado de NPR: https://www.npr. org/sections/goatsandsoda/2020/02/13/805519117/ a-change-in-how-one-chinese-province-reportscoronavirus-adds-thousands-of-cases.

Ferguson, N., Laydon, D., Nedjati-Gilani, G., Imai, N., Ainslie, K., Baguelin, M. ... Ghani, A. C. (2020, 16 de marzo). Impact of non-pharmaceutical interventions (NPIs) to reduce COVID-19 mortality and healthcare demand. Recuperado de Imperial CollegeCOVID-19 Response Team: 
https://www.imperial.ac.uk/media/imperial-college/ medicine/sph/ide/gida-fellowships/Imperial-CollegeCOVID19-NPI-modelling-16-03-2020.pdf

Goodson, M. (2020). All models are wrong. Royal Statitical Society, RSS Data Science Section.

Hume, D. (1981). Tratado de la naturaleza humana. Madrid, España: Orbis.

López, C. (2020, 30 de marzo). Entrevista a Claudia López, Alcaldesa Mayor de Bogotá. (N. Yamid, A., Entrevistador). Recuperado de YouTube: https:// www.YouTube.com/watch?v=kwJyQwTn-1E

Ministerio de Sanidad - Gobierno de España. (2020, 11 de abril). Procedimiento de actuación frente a casos de Infección por el nuevo Coronavirus (SARS - CoV-2). Madrid: Dirección General de Salud Pública, Calidad e Innovación.

Moncada, B. (2020, 26 de Marzo). "Ustedes tienen el tiempo que nosotros no tuvimos en España". Recupeado de Expreso: https://www.Expreso. ec/actualidad/coronavirus-ecuador-carlos-bortestadisticas-contagios-7860.html

Ordway, D. (2020, 26 de marzo). Covering COVID-19 and the coronavirus: 5 tips from a Harvard epidemiology professor. Recuperado de Journalist's Resourse: https://journalistsresource.org/ studies/society/public-health/covid-19-coronavirusepidemiology/

Organización Mundial de la Salud. (2020, 27 de febrero). Global surveillance for COVID-19 caused by human infection with COVID-19 virus. World Health Organization, https://www.who.int/ publications-detail/global-surveillance-for-humaninfection-with-novel-coronavirus-(2019-ncov).

Paullus, J. (2000). Erase una vez un número. Madrid, España: Tusquets Editores S.A.

Red Etica. (2020, 26 de marzo). Coronavirus: 10 virtudes del periodismo responsable ante la pandemia. Recuperado de Red Etica: https://fundaciongabo. org/es/etica-periodistica/recursos/coronavirus10-virtudes-del-periodismo-responsable-ante-lapandemia

Trilla, A. (2020, 01 de febrero). Un mundo, una salud: la epidemia por el nuevo coronavirus COVID-19. Recuperado de Elsevier.es: https://www.elsevier.es/ es-revista-medicina-clinica-2-avance-resumen-unmundo-una-salud-epidemia-S002577532030141X
Wu Z, \& McGoogan JM. (26 de March de 2020). Characteristics of and Important Lessons From the Coronavirus Disease 2019 (COVID-19) Outbreak in China: Summary of a Report of 72314 Cases From the Chinese Center for Disease Control and Prevention. Recuperado de JAMA Network: https://jamanetwork.com/journals/ jama/fullarticle/2762130 